\title{
MỚ RỘNG LớP CÁC SỐ MERSENNE
}

\author{
Lều Dức Tân
}

Ban cơ yéu chính phủ

\section{Mở đầu}

Năm 1640 Mersenne xét lớp các số có dạng $M_{n}=2^{n}-1$ và dề ra giả thuyết rằng: với $n<257$ thì $M_{n}$ là nguyên tố khì và chì khì $n=2,3,5,7,31,67,127$ và 257 .

Guồng máy xác định nhận định trên và hơn nữa đề khảo sát một lớp các số có dạng $M_{n}=2^{n}-1$ với $n$ nguyên tố bắt đầu hoạt động. Các số $M_{2}, M_{3}, M_{5}$ và $M_{7}$ đã được biết là nguyên tố tì thời Euclide. $M_{13}$ cung được biết từ 1461 nhưng không xác định rõ tác giả. Năm 1732 , Euler đã chỉ ra $M_{29}$ là hợp số và năm 1750 chỉ ra $M_{31}$ là nguyên tố. Nhận định cria Mersenne đứng vững hơn 200 năm cho đến khi Lucas chî ra rằng $M_{67}$ là hợp số vào năın 1876. Cho đến 1914 già định của Mersenne mới được bồ xung thên. Ông đà sai trong 5 trường hợp: Thiếu $M_{61}$ (do I.M. Pervushin chì ra 1883 ), $M_{89} M_{10 \tau}$ (do R.E. Powers và E. Fauquenberg chí ra 1914). Và thừa $M_{6 \tau}, M_{25 i}$.

Trong quá trình giải bài toán trên của Mersenne đà hỉnh thành lên một loại kiềm tra tính nguyên tố cho cạ́c số nguyên và được gọi là thuật toán kiêm tra tính nguyên tố kiều- $(N+1)$ như sau:

Gia sư ta biét được khai triên $N+1$ thành tích các thửa số nguyên tố và $D$ là mọt số sao cho $J(D / N)=-1$. Nếu với mối ước nguyên tố p cúa $N+1$, có một dãy Lucas $U_{k}$ với biệt thức $D$ sao cho $N$ là wớc cúa $U_{N+1}$ nhưng không là ước cuia $U_{(N+1) / p}$ thì $N$ là nguyên tố.

Trong phát biêu trên, $J(D / n)$ là ký hiệu Jacobi, còn khái niệm dãy Lucas được định nghía ờ phần 0 . Kết quà tốt nhất vê loại thuật toán này cho bởi định lý sau 
Dịnh lý Lucas. Cho $N=R 2^{k}-1$ với $R$ lé $<2^{k}$ và 3 không lo v̛ớc cúa $R$ và $N$. Khi dó $N$ nguyên tố khi và chì khi $V_{(N+1) / 4} \equiv 0 \bmod N$.

Với kết quả trên không những kiêm tra tính nguyên tố cho các số thuộc lớp các số Mersenne mà còn kiêm tra được cho mọ́ số lớp rộng hơn nhič̀u. Trong bài này chúng tôi sì mờ rộng hơn nữa tầm hiệu lựi "ủa thuật toán kiều $N+1$ nói trên.

Bài báo này nhằm giài quyết vấn đề xây dựng cơ sở lý thuvết cho một thuật toán kiêm tra tính nguyên tố kiều $N+1$ và tư đó tỉm kiếm nhanh cár số ngus în tố lớu trong một lớp số đực ký hiệu là lớp $L M$.

Ta có thề xuất phát tì định lý sau đây.

Dịnh lý Lehmer $([17])$. Cho $N=R F-1,(F, R)=1$. Nếu $\left\{U_{m}\right\}$ là dãy Lucas rới $J(D / N)=-1$ sao cho vói mọi ưóc nguyên tố q của $F$ tı có $U_{N+1} \equiv 0 \bmod N$ và $\left(U_{(N+1) / 2}, N\right)=1$ thì mọi đớc nguyên tố $p$ cir $N$ đều có dạng $p=m F \pm 1$.

Tuy nhiên việc chỉ ra dãy I ucas thoả mãu điều kiện cuả định lý không phài dể dàng, do vậy kết quả khà quan nhất có lính thực hành liên quan đến thuật toán kiểu $N+1$ chi đat đến kết quà của Luca dà nêu ờ trên. Chúng tôi đã tím ra một kết quà (định lạ: 3) tương tự nhưng yếu hơn kết luận cuả Lehmer nhưng dề bù lại chúng tôi đà chỉ ra có tỉnh kiến thiết (điều kiện 7) líp số cụ thề (lớp LM) mà trên đó việc viết chương trỉnh kiêm tra tỉnh nguyên tố "ảc số thuộc lớp đó lại hết sức đơn giản. Ngoài ra chúng tôi đà chúng tỏ được việc tham gia cuả tham số $d$ trong bước kiềm tra tính nguyên tố kiêu $N+1$ vẫn còn có hiệu lực (định lý 4 ) do vậy đã xây dựng được thuật toán $N T$ mà lợp các số kiêm tra được cuả nó lớn hơn hẳn lớp các số kiềm tra được cuà thuật toán cuà Lucas về bậc.

Nội dung cuà báo gồm crác phần sau:

Phận 0 nhằm giới thiệu về khái niệm dày Lucas và các kết quả liên quan đến việc chứng minh các kết quả ờ các phần sau.

Trong phần 1 chưng tôi chứng minh thêm một số tính chất khác cần cho mục dích về sau.

Phần 2 bao gồm các kết quà chính để xây dựng một lớp số và một thuật toán đa thức đề kiêm tra tính nguyên tố đối với các số thuộc lớp đó.

Trong phần 3 chúng tôi đưa ra kết quà tính toán thu được khi thực hiện tím kiếm các nguyên tố bằng thuật toán xây dụ̣ng được ờ phần trên.

\section{Các khái niệm và kết quả liên quan}

\subsection{Kýy hiệu Legendre và ký hiệu Jacobi}

Cho $p$ nguyên tố và $a$ là số nguyên tố đối với $p$, khi đó số

$$
L(a / p)= \begin{cases}1, & \text { nếu có } b \text { sao cho } a \equiv b^{2} \bmod p \\ -1, & \text { trong trường hợp ngược lại }\end{cases}
$$


được gọi là ký hiệu Legendre cúa $a$ và $p$.

Dịnh nghia 0.2. Cho $m, n$ nguyêu với $n>3,(m, n)=1$. Nếu $n=p_{1} p_{2} \ldots p_{r}$ trong dó $p_{i}$ là các nguyên tố (không uhất thiết khác nhau) ta gọi $/(\mathrm{m} / \mathrm{n})=L\left(\mathrm{~m} / \mathrm{p}_{1}\right) L\left(\mathrm{~m} / \mathrm{p}_{2}\right) \ldots L\left(\mathrm{~m} / \mathrm{p}_{\mathrm{r}}\right)$ là ký hiệu lacobi cuia $m$ và $n$.

Dịnh lý 0.3 (luật bỉnh phırơng tương hồ). Cho các số nguyên lé $m, n$ với $(m, n)=1$, khi đó

$$
J(m / n)=(-1)^{1 / 4(M-1)(N-1)} J(n / m) .
$$

Dịnh lý 0.4. Nếu p là nguyên tố lí khi dó crị mọi a mà $(a, p)=1$ ta có $J(a / b)=$ $L(a / p)=\operatorname{amod} p$.

Các kết quả trên có thề tìm trong [1].

\section{Các kết quá trên trường $Z_{F}(\sqrt{D})$}

('ho $p$ nguyên tố khi đó vành dồng du modulo $p$, kỵ hiệu là $Z_{p}$, là trường hữu hạn $p$ phần tì̛. Nếu $d$ là số sao cho $p$ không là ước cuà $I)$ ta ký hiệu

$$
Z_{p}(\sqrt{D})=\left\{x+y \sqrt{D} \mid x, y \in Z_{p}\right\}
$$

và xây dụng các phép toán trên dó như sau:

Nếu $a_{1}=x_{1}+y_{1} \sqrt{D}$ và $a_{2}=x_{2}+y_{2} \sqrt{D}$ ta gọi tồng $a_{1}$ và $a_{2}$ là phần tử ký hiệu $a_{1}+a_{2}$ xác định bời công thức

$$
a_{1}+a_{2}=\left(x_{1}+x_{2}\right)\left(y_{1}+y_{2}\right) \sqrt{D}
$$

và tích của $a_{1}$ và $a_{2}$ là phần tủ kị́ hị̣̂̂ là $a_{1} \cdot a_{2}=\left(x_{1} x_{2}+y_{1} y_{2} D\right)+\left(x_{1} y_{2}+x_{2} y_{1}\right) \sqrt{D}$

Kết quà 0.5 . Tập $Z_{p}(\sqrt{D})$ rới các phép toán cọng và uhân xây dựng ó trên là trường. Hơn nữa nếu $J(D / p)=1$ thì $Z_{p}(\sqrt{D}) \equiv Z_{p}$ còn nếu $\left.J(I) / p\right)=-1$ thì $Z_{p}(\sqrt{D})$ là trường mờ rộng cuà $Z_{p}$ với số phần tì̛ là $p$.

Dịnh lý 0.6. ( Định lý Fermat trên $Z_{p}(\sqrt{D})$. I'ói $a \neq 0$ và $a \in Z_{p}(\sqrt{D})$ ta có

$$
\begin{aligned}
& \text { (a) } a^{p-1} \equiv 1 \bmod p \text { hhi }(D / p)=1, \\
& \text { (b) } a^{p} \equiv a^{*} \bmod \operatorname{khi}(D / p)=-1
\end{aligned}
$$

ó đây $a^{*}=x-y \sqrt{l}$ ) (với $a=x+y \sqrt{D}$ ) và gọi là liên hợp cuáa

Các kết quà trên có thê tìm trong [15] hay [17]. 


\subsection{Dãy Lucas}

Cho phương trình với hệ số $P, Q$ nguyên

$$
\lambda^{2}-P \lambda+Q=0 .
$$

Giả sử $a$ và $b$ là hai nghiệm của phương trình (1) với $m \geq 0$ ta ký hiệu

$$
U_{m}=\left(a^{m}-b^{m}\right) /(a-b) \text { và̀ } V_{m}=a^{m}+b^{m} .
$$

Dịnh nghĩa 0.7 . Dãy các số $\left\{U_{m}\right\},\left\{V_{m}\right\}$ gọi là dãy Lucas cuả phương trình $(1)$ và phương trình (1) gọi là phương trình đặc trưng cuả dãy Lucas $\left\{U_{m}\right\},\left\{V_{m}\right\}$ với $U_{m}$, $V_{m}$ xác dịnh ờ công thức (2).

Ta thấy rằng $U_{0}=0, U_{1}=1, V_{0}=2, V_{1}=P$ là các số niguyên.

Công thức 0.8. Với $m \geq n \geq 0$ ta có

$$
\left\{\begin{array}{l}
U_{m+n}=U_{m} V_{n}-Q^{n} U_{m-n} \\
V_{m+n}=V_{m} V_{n}-Q^{n} V_{m-n}
\end{array}\right.
$$

Trường hợp $n=1$

$$
\left\{\begin{array}{l}
U_{m+n}=P U_{m}-Q U_{m-1} \\
V_{m+n}=P V_{m}-Q^{n} V_{m-1}
\end{array}\right.
$$

Trường hợp $m=n$

$$
\left\{\begin{array}{l}
U_{2 m n}=U_{m} V_{m} \\
V_{2 m}=V_{2 m}-2 Q^{m}
\end{array}\right.
$$

Trường hợp $m=n+1$.

$$
\left\{\begin{array}{l}
U_{2 m-1}=U_{m} V_{m-1}-Q^{m-1} \\
V_{2 m-1}=V_{m} V_{m-1}-P Q^{m-1}
\end{array}\right.
$$

Tính chất 0.9. Cho dãy Lucas $\left\{U_{m}\right\}$ khi đó néu i là v̛ớc cuà $j$ thi $U_{i}$ là ước cuà $U_{j}$. Tính chất 0.10 . V'ó $i$ mọi $M \geq 0$ ta có

$$
\text { - }\left[\begin{array}{cc}
U_{m+1} & V_{m+1} \\
U_{m}^{\prime} & V_{m}
\end{array}\right]=\left[\begin{array}{cc}
P & -Q \\
1 & 0
\end{array}\right]^{m}\left[\begin{array}{cc}
U_{1} & V_{1} \\
U_{0} & V 0
\end{array}\right] .
$$

Tính chất 0.11. Cho $\left\{U_{m}\right\}$ là dãy Lucas cua phương trinh $\lambda^{2}-P \lambda+Q=0$ với biệt thức $P^{2}-4 Q=x^{2} D$ trong đó $D$ không có thừa số chính phương. Khi đó nếu số nguyên tố $p$ không là ước cuả $D . Q$ thì $U_{p-J(D / p)} \equiv 0 \bmod p$.

Diều kiện Riesel 0.12. Phương trinh dặc trưng $\lambda^{2}-P \lambda+1=0$ với biệt thức $P^{2}-4=x^{2} D$ trong đó $J(D / N)=-1$ và $D$ không có thừa số chính phuơng, có nghiệm a $=b^{2} / r$ sao cho

$$
\left.b b^{*} / r\right) J(r / N)=-1,
$$

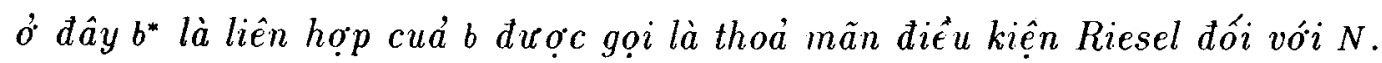


Dịnh lý 0.13 (xem [17]) Cho $. v=R 2^{k-1}-1$ vói $R$ lé $R \leq 2^{k}$ ià $\left\{V_{m}\right\}$ là dãy Lucas cuá phưong trinh thoả mãn diể kiện Riesel đối với $N$. Khi đó 2 diểu kiện sau là tương duong:

(a) $N$ nguyên tó

(b) $V_{(N+1) / 4} \equiv 0 \bmod N$.

\section{Các kết quả lý thuyết}

\subsection{Bồ rung về tính chất cuả dãy Lucas}

Từ tính chất 0.11 ta có quyền định nghià như sau:

Dịnh nghiã 1.1. Ta gọi giá trị $d=\min _{m>0}\left\{U_{m} \equiv 0 \bmod p\right\}$ với $p$ nguyên tố không là ước cuả $D . Q$ là bậc cuả $\left\{U_{m}\right\}$ dối với $p$, ờ đây $\left\{U_{m}\right\}$ là dãy Lucas cuà phương trỉnh đặc trưng $\lambda^{2}-P \lambda+Q$.

Ta có tính chất sau đày.

Tính chất 1.2. Néu d là bộc cuni $\left\{U_{m}\right\}$ đối với $p$ và nếu $U_{m} \equiv 0$ mod $p$ thì d là uớc r uả $m$.

Chứng minh. Già sừ

$$
m=q d+r \text { với } 0 \leq r \leq d .
$$

Trường hợp $q=2 t-1$ (tr. 1)

$$
\begin{aligned}
U_{m} & =U_{t d+\{(t-1) d+r}^{r} \\
& =U_{t d} V_{(t-1) d+r}-Q^{(t-1) d+r} U_{d-r} .
\end{aligned}
$$

Tì dịnh nghià 1 ta có $p$ là ước cuả $U_{d}$ mà $d$ là ước cuà $t d$ nên theo tính chất 0.9 ta có $U_{d}$ là ước cuả $U_{t d}$ nên $p$ là ước cuà $U_{t d}$ mặt khác $p$ là vớc ruà

$$
U_{m}==U_{t d} V_{(t-1) d+r}-Q^{(t-1) d+r} U_{d-r},
$$

do dó ta có $p$ là ước cuà $Q^{(t-1) d+r} U_{t-r}$ mà $p$ không là ước cuà $Q$ vậy $p$ là ước cuà $U_{d-r}$.

Do $d$ là bé nhất nên $d \leq d-r$ hay $r=0$.

Trường hợp $q=2 t$

Rõ ràng ta chỉ cấn xét với $t \geq 1$ và như vậy $m=q d+r=2 t d+r=(t+1) d+(t-1) d+r \quad$ ! $+r$ nên $U_{m}=U_{(t+1) d+(t-1) d+r}$ theo công thức 0.8 [3] thì

$$
U_{(t+1) d+(t-1) d+r}=U_{(t+1) d} V_{(t-1) d+r}-Q^{(t-1) d+r} U_{2 d+r} .
$$

Lập luận như trường hợp trên ta có $U_{2 d-r} \equiv \bmod p$. Như vậy lại từ công thức 0.8 $[3]$ ta có

$$
0 \bmod p \equiv U_{2 d-r}=U_{d+d-r}=U_{d} V_{d-r}-Q^{d-r} U_{r}
$$

cho nên $U_{r} \equiv 0 \bmod p$ mà $d$ bé nhất và theo [8] thì $r<d$ cho nên $r=0$. Tóm lại ta đêu có $m=q d$ hay $d$ là ước cuà $m$. 
1.2. Kêt quà chinh

Dịnh lý 1.3. Cho, $v=R \cdot 2^{k}-1$ với $R$ lé. Cho $\left\{V_{m}\right\}$ là dày Lucrs cua phưong trinh đăc trung

$$
\lambda^{2}-P \lambda+1=0
$$

thoả mãn điêu kiện Rie.el đối với $N$. Khi dó ta có

(a) Néu $\Lambda$ nguyén tố thi $V_{(N+1) / 4} \equiv 0 \bmod v$.

(b) Néu $V_{(N+1) / 4} \equiv 0 \bmod \Lambda$ thî mọi ước nguyên tố p cuả $N$ dểu có dạng $p=s 2^{k-1} \pm 1$.

Chứng minh. Trước hết do hệ số $Q$ cuà phương trỉnh dặc trưng bằng 1 nên ta có tích hai nghiệm cuà phương trỉnh (10) bằng 1 vậy nếu a là nghiệm cuả phương trỉnh (10) thi $a^{*}$ cùng là nghiệm và do dó bằng liên hợp cuá $a$.

Chưng minh (a)

$$
\begin{aligned}
V_{(N+1) / 4} & =a^{(N+1) / 4}+a^{-(N+1) / 4} \\
& =a^{-(N+1) / 4}\left(a^{(N+1) / 2}+1\right) \text { mà } a=b^{2} / r \text { nên } \\
& \left.=a^{-(N+1) / 4}\left(b^{N+1}\right) / r^{(N-1) / 2}+1\right) \\
& \left.=a^{-(N+1) / 4}\left(b b^{N}\right) r / r^{(N-1) / 2}+1\right),
\end{aligned}
$$

do $N$ nguyên tố theo các định lý 0.6 ta có $b^{*} \equiv b^{*} \bmod N$ (với $b^{*}$ là liên hợp cuà $b$ ) và theo dịnh lý 0.4 ta có $r^{(v-1) / 2} \equiv J(r / N) \bmod N$ vậy

$$
l_{(N+1) / 4}=a^{-(N+1) / 4}\left(b b^{*} / r J(r / N)+1\right)
$$

tù điều kiện Riesel ta có $b b^{*} / r r^{(N-1) / 2}=-1 n \operatorname{nện} b b^{*} / r r^{(N-1) / 2}+1=0$ hay $V_{(N+1) / 4} \equiv$ $0 \bmod N$

\section{Chüng minh (b)}

Tir $V_{(N+1) / 4} \equiv 0 \bmod N$ theo lập luận trên ta có

$$
b b^{*} / r r^{(N-1) / 2}=-1 \bmod N \text {. }
$$

Mặt khác theo công thức 0.8 [5] ta có

$$
U_{(N+1) / 2}=U_{(N+1) / 4} V_{(N+1) / 4} \equiv 0 \bmod N
$$

Theo định nghià cuả dãy Lucas ta có

$$
U_{(N+1) / 4}=\left(a^{(N+1) / 4}-a^{-(N+1) / 4}\right) /\left(a-a^{-1}\right) .
$$

Lập luận như phần trên thì

$$
U_{(N+1) / 4}=a^{-(N+1) / 4}\left(b b^{*} / r r^{(N-1) / 2}-1\right) /\left(a-a^{-1}\right) \equiv\left(a^{(N+1) / 4}-a^{-(N+1) / 4}\right) /\left(a-a^{-1}\right) \bmod N,
$$


tự $N$ lè nîn $(-2, N)=1$ còn $a^{(N+1) / 4}$ và $a-a^{-1}$ là các phần từ có ngược trong $Z_{N}(\sqrt{D})$ nên ta có

$$
U_{(N+1) / 4} \not \equiv 0 \text { modulo } N \text {. }
$$

Nếu $p$ là ước nguyên tố cuà $N$ thì tì̀ các hẹ thức (12) và (13) ta có

$$
\begin{aligned}
& U_{(N+1) / 2} \equiv 0 \bmod 9 \\
& U_{(N+1) / 4} \not \equiv 0 \operatorname{modulo} p .
\end{aligned}
$$

Như vậy nếu $d$ là bậc cuà $U_{m}$ đối với $p$ tù $(14)$ và $(N+1) / 4=R 2^{k-2}$ vậy $2^{k-1}$ là ước cuá $d$. Lại theo định lý 0.11 ta có $U_{p-L(D / p)} \equiv 0 \bmod p$ theo tính chất 2 thì $d$ là ước cuả $p-L(D / p)$ do đó $2^{k-1}$ cũng là ước cuả $p-L(D / P)$ và ta có ngay

$$
p=s 2^{k-1}+L(D / p)=s 2^{k-1} \pm 1 .
$$

\subsection{Các thìca số dạng $M 2^{k} \pm 1$}

Dịnh lý 1.3 đã cho chúng ta nhiều hơn một điều kiện cân cho một số là nguyèn tố. Dề đạt dược thuật toán cần thiết chúng ta chứng minh định lý sạ đây

Dịnh lý 1.4. Cho $N=A q^{2}+B q-1$ với $q$ chẵn. $0<B<q, A>1$. Khi đó các điều kiện sau là tuong dương:

(a)

$$
N=(x q+1)(y q-1) \text { với } x, y \geq 1
$$

(b) tồn tại d thoá mãm $d_{1} \leq d \leq d_{2}$ với $d_{1}=(-B-A+1) \operatorname{din}(q-1)$ còn $d_{0}=0$ nêu $A-B-1<0$ và bằng $(A-B-1) \operatorname{div}(q+1)$ nếu $A-B-1 \geq 0$ [17] sao cho $(B+d q)^{2}+4(A-d)$ chính phuoong [18].

Chứng minh. (a) $\Longrightarrow$ (b). Tì̀ $N=(x q+1)(u q-1)=x y q^{2}+(y-x) q-1$ ta có $B=$ $(y-x) \bmod q$ và $A=x y+(y-x) \operatorname{div} q$. Dặt $d=(y-x) \operatorname{div} q$ ta có $y-x=B+d q$ và $y x=A-d$. Vậy

$$
(B+d q)^{2}+4(A-d)=(y+x)^{2}
$$

tức (18) được thoà mãn.

Trường hợp $d \geq 0$ thì $B+d q \geq 0$ vậy

$$
1 \leq \min (x, y)=0.5\left(\sqrt{(B+d q)^{2}+4(A-d)}-(B+d q)\right)
$$

ta có

$$
\begin{aligned}
(B+d q)^{2}+4(A-d) & \geq(B+d q+2)^{2} \\
4(A-d) & =4(B+d q)+4 \\
A-B-1 & \leq d(q+1) \text { hay } \\
d & \leq(A-B-1) \operatorname{div}(g+1)
\end{aligned}
$$


Trương hợp $d<0$ thì $B+d q<0$ vậy

$$
1 \leq \min (x, y)=0.5\left(\sqrt{(B+d q)^{2}+4(A-d)}+(B+d q)\right)
$$

ta có

$$
\begin{aligned}
(B+d q)^{2}+4(A-d) & \geq(2-(B+d q))^{2} \\
4(A-d) & =4-4(B+d q) \\
d(q-1) & \leq-A-B+1 \text { hay } \\
d & \leq(-A-B+1) \operatorname{div}(g+1) .
\end{aligned}
$$

Kết hợp hai trường hợp trên ta có (17) được thoả mãn.

(b) $\Longrightarrow$ (a). Tì $d$ thoà màn $(18)(B+d q)^{2}+4(A-d)=C^{2}$ khôn! giàm tồng quát giả sư $C \geq 0$. tta đặt $x=0.5(C-(B+d q))$ và $y=0.5(C+(B+d q))$ khi đó rõ ràng ti ó $x, y \geq 0$ và

$$
\begin{aligned}
(x q+1)(y d-1) & =x y q^{2}+(y-x) q-1 \\
& ==0.25\left(C^{2}-(B+d q)^{2}\right) q^{2}+0.5(2(B+d q)) q-1 \\
& =0.25(1(A-d)) q^{2}+B q+d q^{2}-1 \\
& =A q^{2}+B q-1 \\
& =N .
\end{aligned}
$$

Mặt khác tiù điều kiện (17)

$$
(-B-A+1) \operatorname{div}(q-1) \leq d \leq(A-B-1) \operatorname{div}(q+1) \cdot
$$

ta có nếu $d<0$ thì $y \leq x$ và

$$
y=\min (x, y)==0.5\left(\sqrt{(B+d q)^{2}+4(A-d)}+(B+d q)\right)
$$

mà

$$
\begin{aligned}
d & \geq(-A-B+1) \operatorname{div}(q-1) \\
d(q-1) & \geq-A+B+1 \\
4(A-d) & \geq 4-4(B+d q) \\
(B+d q)^{2}+(A-d) & \geq(2-(B+d q))^{2} \\
\sqrt{(B+d q)^{2}+4(A-d)}+(B+d q) & \geq 2
\end{aligned}
$$

đo đó $x, y \geq 1$. Trường hợp $d \geq 0$ ta cũng có lập luận tương tự và như vậy định lý đã được chứng minh.

Đến đây ta cùng rút ra một kết quả cuói cùng như sau: 
Dinh lý 1.5 . Cho

(i) $N=A 2^{2 k}+B 2^{k}-1$, vó ik $>2,2 \| B$ và $0<B<2^{k}$.

(ii) $\left\{V_{m}\right\}$ là dây Lucos cuả phuóng trinh $x^{2}-P \lambda+1=0$ thoá mãn diều kiện Riesel đối với $N$.

Whi đó các điều kiện sau là tương dương

(a) $N$ nguyên tố.

(b) $N$ thou mãn các điêu kiện sau:

(*) $V_{(N+1) / 4} \equiv 0 \bmod N$

$\left(^{* *}\right)$ Nêu $A>0$ thi với mọi d thoá mãn $d_{1} \leq d \leq d_{2}$ với $d_{1}=(-B-A+1) \operatorname{div}(2-1)$ còn $d_{2}=0$ nếu $A-B-1<0$ và bằng $(A-B-1)$ div $\left(2^{k}+1\right)$ nếu ngược lại thì $(B+d q)^{2}+4(A-d)$ không chinh phương.

Chứng minh. (a) $\Longrightarrow(\mathrm{b})$.

Nếu $N$ nguyên tố từ định lý 3 ta có $\left({ }^{*}\right)$ thoả mân, giả sư $\left({ }^{* *}\right.$, không thuả mãn thì từ định lỵ 4 thì $N$ phài là hợp số. Diều vô lý trền dẫn đến $\left(^{* *}\right)$ phải được thoà màn.

(b) $\Longrightarrow(\mathrm{a})$. Tì̛ giả thiết điêu kiện Riesel thoả mãn cùng với các điểu kiện $\left(^{*}\right)$ nên giả thiết cuà định lý 3 được thoà mãn nên ta có mọi ước nguyên tố cuà $N$ và do dó mọi ước cuả $N$ đều có dạng $s 2^{k}+1$. Như vậy nếu $N$ là hợp số thì $N=U V$ với $U=s 2^{k} \pm 1, V=s^{\prime} 2^{k}-( \pm) 1$ với $s, \grave{s}^{\prime} \geq 1$, lại theo định lý 4 ta có $d$ thoà mãn $d_{1} \leq d \leq d_{2}$ sao cho $\left(B+d 2^{k}\right)+4(A-d)$ chính phương, điều này mâu thuấn với điều kiện $\left(^{*}\right)$ và do đó định lý đà được chứng minh.

\section{Thuật toán kiểm tra tỉnh nguyên tố kiều $N+1$}

\subsection{Thuật toán.}

Định lý 1.5 trên là cơ sờ thuật toán sau.

Thuật toán NT. Dê kiêm tra tính nguyên tố cuà số $N$ ta tiến hành qua các bước : sau:

Bước 1: Biêu diễn $N=A 2^{2 k}+B 2^{k}-1$, với $2 \| B, 0<B<2^{k}$.

Bước 2: Tìm phương trình trong điều kiện (ii) cuà định lý 5 .

Bước 3: Kiêm tra điều kiện $V_{(N+1) / 4} \equiv \bmod N$.

$\left(^{*}\right)$ Nếu sai kết luận $N$ là hợp số, dùng chương trình

$\left(^{*}\right)$ Ngược lại kiêm tra điều kiện $A=0$.

+ Nếu đúng kết luận $N$ là nguyên tố, dùng chương trình.

+ Ngược lại sang bước 4.

Bước 4: Lần lượt kiềm tra tính chính phương cuà $S(d)=\left(B^{\prime}+d 2^{k}-1\right)^{2}+4\left(A^{\prime}-d\right)$ với $d$ lần li̛ợt tì $d_{1}$ đến $d_{2}$ với $d_{1}, d_{2}$ như trong định lý 1.5 .

* Nếu đúng với một $d$ nào đó thì kết luận $N$ là hợp số, dùng chương trình.

* Ngược lại kết luận $N$ là nguyên tố, dùng chương trình. 
44

Nhận xét: Về nguyên tắc thì thuật toán nêu ở trên có thể kiêm tra tỉnh nguyền tố cho một số lé mà với nó tồn tại phương trỉnh thoà mãn Riesel. Chúng tôi sẽ chi ra các lớp số mà với chúng việc tỉm phırơng trỉnh thoả mãn điểu kiện này có thề bằng cách tra bàng (điều kiện) tuy nhî̂n tînh đa thức cuả nó vẫn không đạt được do bước 4 . Ở đây chúng tôi sê đưa ra một lớp số cụ thề mà trên đó thuật toán NT dùng kiềm tra tíuh nguyên tố các số thuộc lớp đó sè đạt tính đa thức.

\subsection{Việc hạn chê lớp số}

Đề thuật toán NT có thê thực hiện trong thời gian đa thức ta huộc phải hạn chế đối tượng đàu vào, việc hạı chế cụ thề được trỉnh bày sau đây.

Đối với khó khărn gây ra bời bước 2 ta chi xét lớp các số mà với chúng việc lìm phương trỉnh đặc trưng thoà mãn điêu kiện Riesel có thể thấy ngay. Sau đầ sć đưa ra vài lớp số mà với chúng tôi cần thông qua một kiêm tra dơn giản ta có i hể tìm được phương trỉnh thoà màn điều kiện Riesel hằng cách tra bảng.

Diều kiện $\left(^{*}\right)$. Cho $N$ có $J(D / N)=-1$. Khi đó phương trình dặc trưng $\lambda^{2}-P \lambda+1=0$ thoả mãn điền kiện Riesel có thề tim được qua bảng sau

Bảng 2: Hệ số và nghiệm cuả phương trình thoả mãn điêu kiện Riesel

$\begin{array}{lllll}\mathrm{D} & 4 & \text { nghiệm phroong trình } & \mathrm{b} & \mathrm{r} \\ 3 & 4 & 2+\sqrt{3} & 1+\sqrt{3} & 2 \\ 5 & 3 & 0.5(3+\sqrt{5}) & 1+\sqrt{5} & 4 \\ 11 & 22 & 10+3 \sqrt{11} & 3+\sqrt{11} & 2 \\ 13 & 11 & 11+3 \sqrt{13} & 3+\sqrt{13} & 4 \\ 17 & 66 & 33+8 \sqrt{17} & +1+\sqrt{17} & 1 \\ 19 & 340 & 170+39 \sqrt{19} & 13+3 \sqrt{19} & 2 \\ 29 & 27 & 0.5(27+\sqrt{29}) & 5+\sqrt{29} & 4\end{array}$

Tất nhiên ta có thề làm tăng số lượng cuà bảng trên bằng cách dò tìm nhưng ờ đây cũng nảy sinh vấn đề nà bài báo này chưa giải quyết được đó là:

Liệu có hay không phương trình thoá mân điêu kiĉ̣n Rirsel dối với số $\mathrm{v}$ cho trước?

Tuy nhiên, ta có thề ta có thề suy ra được kết quà sau:

Dịnh nghiã 2.1. $L M=\{N$ lè: tồn tại $a \in[3 ; 5 ; 11 ; 13 ; 17 ; 19 ; 29]$ sao cho $J(a / N)=-1$ và $R \leq c^{\prime} k^{2}$ với $\left.N=R 2^{k}-1\right\}$.

Từ đó ta có: 
Dịnh lý 2.2. Thuật toán NT là thuậl toán kị̂̂m tiu nhanh tính nguyên tố các số lớp $L M$.

\section{Phần 3. Dánh giá về thuật toán $N T: 3$ và lớp số $L M$.}

Hoàn toàn tương tự như ở mục 3 chương trước ta cũng thu được các kết luận sau:

3.1. Dánh giá về lớp kicin tra được

Theo định lý Lucas ta thấy rằng lớp kiêm tra được cuả thuạt toán $N T$ là rộng hợ rất nhiều. Nếu như trong định lý Lucas yêu cầu đối với $N$ phải là $\leq 2^{2 k}$ thì ớ thuật toán $6, N$ thậm chí có thề $>2^{3 k}$ với $N=R 2^{k}-1, R$ lè. Đây là bước tiến khá lớn về mặt phát huy khả năng cuà các thuật toán kiều $N+1$. Sau đây ta sẽ phân tích cụ thể một lớp con cuả lớp các số kiêm tra được cuả thuật toán $N T$.

Kìy hiệu:

$$
M s=\{N=12 m-5 \mid m l \dot{\mathrm{e}}\}
$$

và

$$
M s(k)=\left\{N=R 2^{k}-1 \mid R \text { lè } k>2 \text { và }(-1)^{k} R=-1 \bmod 3\right\}
$$

ta dễ dàng nhận được các kết quả sau:

Kết quá 3.1. $\left\{M_{3}(k)\right\}, k>2$ là phân hoạch cuà $M_{3}$.

Kết quá 3.2. Hai phần tử kề nhau trong $M_{3}(k)$ cách uhau là $6.2^{k}$.

Ký hị̣̂u $L M_{3}=\{N \in L M \mid J(3 / N)=-1\}$, ta có kết quả sau:

Kết quả 3.3. Cho trước số $x>0$ khi đó số các số $N$ thuộc lớp $L M_{3}$ không quá $x(N \leq x)$ là $O\left(x^{2 / 3}\right)$.

\subsection{Thời gian tính cuả thuật toán $N T$}

Người ta đà chì ra rằng thời gian tính cuà thư tục tìm số Lucas thứ $M$ là $\mathrm{O}\left(\mathrm{m}^{5}\right)$ và xác định tỉnh chính phương cuả số $M$ có thơi gian tính là $O\left(m^{3}\right)$ với $m=\log M$. Trong thuật toán $N T$ ta chì cần một lần tỉm số Lucas thứ $(N+1) / 4$ và dối với $N \in L M$ thì ta cần không quá $C$ lần xác định tỉnh chính phương cuả $S(d)$. Do vậy ta có

Dịnh lý 3.4. Thuật toán NT thực hiện trên lóp LM có thời gian tính là $O(n)$, ơ đây $n=\log N$, với $N$ là đầu vào.

\subsection{Kết quà thục hành}

Chúng tôi đã thể hiện thuật toán trên trong một bộ chương trỉnh viết bằng ngôn ngữ TUBO-PASCAL, các kết quả thu được khi chạy chương trỉnh như sau: 
+ Đã tìm trong 95 lớp các số $L M_{3}(k), k=3 \ldots 96$ và thấy rằng

* Luân tồn tại số nguyên tố trong các lớp đó.

* Có 21 lớp ngay số thứ nhất là nguyên tố.

* Có 55 lớp có số nguyên tố troug 9 số đầu cuả lớp

* Và lớp có số nguyên tố dâu tiên xa nhất là lớp $L V_{3}(51)$, nó là số thứ 70 cuà lớp.

Bảng 3. Số thí̛ tụ cuả số nguyên tố đầu tiên cuả các lớp $L M_{3}(k)$ với $k$ từ 3 cho đến 95 .

$\begin{array}{llllllllllll}\mathrm{k} & \mathrm{t} & \mathrm{k} & \mathrm{t} & \mathrm{k} & \mathrm{l} & \mathrm{k} & \mathrm{t} & \mathrm{k} & \mathrm{t} & \mathrm{k} & \mathrm{t} \\ 3 & 1 & 19 & 1 & 35 & 5 & 51 & 70 & 67 & 8 & 83 & 16 \\ 4 & 1 & 20 & 3 & 36 & 3 & 52 & 11 & 68 & 30 & 84 & 15 \\ 5 & 1 & 21 & 2 & 37 & 6 & 53 & 32 & 69 & 10 & 85 & 31 \\ 6 & 3 & 22 & 11 & 38 & 2 & 54 & 1 & 70 & 5 & 86 & 51 \\ 7 & 11 & 23 & 3 & 39 & 5 & 55 & 9 & 71 & 13 & 87 & 33 \\ 8 & 1 & 24 & 14 & 40 & 18 & 56 & 54 & 72 & 1 & 88 & 24 \\ 9 & 2 & 25 & 11 & 41 & 4 & 57 & 10 & 73 & 16 & 89 & 1 \\ 10 & 1 & 26 & 2 & 42 & 9 & 58 & 13 & 74 & 19 & 90 & 18 \\ 11 & 5 & 27 & 21 & 43 & 14 & 59 & 20 & 75 & 5 & 91 & 21 \\ 12 & 1 & 28 & 11 & 44 & 6 & 60 & 3 & 76 & 5 & 92 & 40 \\ 13 & 1 & 29 & 2 & 45 & 2 & 61 & 1 & 77 & 10 & 93 & 49 \\ 14 & 1 & 30 & 7 & 46 & 4 & 62 & 9 & 78 & 8 & 94 & 11 \\ 15 & 9 & 31 & 1 & 47 & 6 & 63 & 11 & 79 & 13 & 95 & 9 \\ 16 & 3 & 32 & 1 & 48 & 1 & 64 & 13 & 80 & 19 & & \\ 17 & 1 & 33 & 6 & 49 & 4 & 65 & 12 & 81 & 9 & & \\ 18 & 1 & 31 & 21 & 50 & 2 & 66 & 16 & 82 & 24 & & \end{array}$

+ Đã kiềm tra 817 số ngầu nhiên > 100 clıữ số thập phân trong lớp $L M_{3}$ và thu được

* 10 số nguyên tố.

* Trong đó có 131 hợp số được phát hiện bời việc kiêm tra $V_{(N+1) / 4} \equiv \bmod N$.

* Không có hợp số nào bị phát hiện bời lọc chính phương.

+ Đã kiềm tra 306 mầu số ngẩu nhiên có đúng 151 chữ số thập phân trong lớp $L M$ và thu được

* 2 số nguyên tố.

* trong đó có 33 hợp số được phát hiện bời việc kiềm tra $V_{(N+1) / 4} \equiv 0 \bmod N$.

* không có hợp số nào thoả mãn $V_{(N+1) / 4} \equiv \bmod N$ nhưng bị phát hiện bới lọc chính phương. 
+ Đã kiêm tra 783 số ngẫu nhiên có 300 chữ số thập phân trong lớp $L M$ và thu được

* 2 số nguyên tố.

* trong đó có 83 hợp số được phát hiện bởi việc kiêm tra $V_{(N+1) / 4} \equiv \bmod N$.

* không có hợp số nào thoà mãn $V_{(N+1) / 4} \equiv 0 \bmod N$ nhưng bị phát hiện bởi lọc chính phương.

\section{Kết luận}

Mặc dù các kết quả thu được về thuật toán kiều $N+1$ cũng như các số kiêm tra được (lớp $L M$ ) cuả chúng tôi đưa ra trong bài báo này vẫn còn bị hạn chế rất nhiêu nhưng dù sao với các kết quà th" được từ thực hành chúng ta hy vọng những kết quả trên sẽ cung cấp cho chúng ta một khả năng tạo lập nhanh kho số nguyên tố lớn.

Lời cám ơn. Các kết quả trên đà đı̛ợc trình bày tại các xê mi na cuả Viện toán học và cuả bộ môn Toán học Viện kỹ thuật quân sự. Tác già xin chân thành cảm ơn các thành viên tham gia về những ý kiến nhận xét quý báu giúp cho tác giả hoàn thiện các kết quả đạt được.

\section{Tài liệu tham khaó}

1. Allency L.B.J.T. and Redfern E.J., Introduction to Number Theory with Computing, Hodder and Shoughton, 1989.

2. Bach F., Bounds for Primality Testing and related Problems, Math. Comp. 55. 1990, n. 191.

3. Pomerance C., Cryptology and Computational Number Theory, Proc. Symp.' Appl. Math. 42, 1990.

4. Erdos P. and Pomerance C., On the number of False Witnesses for a Composite Number, Math. Comp. 46, 1986.

5. Guy R.K., Lacampagne C.B. and Selfridge J.L., Primes at Glance, Math. Comp. 48, 1987, 177.

6. Goldwasser S. and Kilian J., Almost all primes can be quickly certified, Laboratory for Computer Science Massachusetts Institute of Technology, 1986.

7. Ireland K. and Rosen M., A classical introduction to Morden number theory, Springer, 1982.

8. Su Hee Kim and Pomerance C., The probability that a random probable prime is composite, Math. Comp. 53, 1989, 188.

9. Kranakis E., Primality and Cryptography, John Wiley and Sons, 1986.

10. Kurtz G.C., Shanks D. and Williams H.C., Fast primality test for numbers less than $50.10^{9}$, Math. Comp., 46 19ז3, 179.

11. Lidl R. and Niederreiter H. , Fin te ficlds, Addison-Wesley, 1983. 
. 12. Parady B.K., Smith J.F. and Zarantonello S., Largest know twin primes, Math. Comp. 55, 1990, 191.

13. Pomerance C., Very Short primality proof, Math. Comp. 48, 1987, 177.

14. Pintz J., Williams L.S. and Szemeredi E., Infinite Sets of Primes with fast primality tests and quick generation of large primes, Math. Comp. 53, 1989, 157.

15. Pratcbar K., Verteilung der Prizahlen, Springer, 1957.

16. Ribenboim P., The litllc book of big primes, 1991.

17. Riesel H., Prime number and computer methods for factorization, Progress in Math. 57, 1985.

18. Salomaa A., Public-key cryptography, EATCS, 1990.

19. Williams H.C., Primality testing on a computer, Ars Combin, 5, 1978.

20. Williams H.C. and Zarnke C.R., Some prime numbers of the forms $2 A 3^{n}+1$ and $2 A 3^{n}-1$, Math. Comp. 26, 1972, 120.

\section{Abstract}

Extension of class of Mersenn's numbers

In this paper we investigate a class of nature numbers denoted by LM which properly includes the class of Mersenn ones. For this class, we propose an algorithm. for testing primality. The algorithm works in polynomial time according to input belonging to $L M$.

The problem that whether or not LM contains infinitely-many primes, even under the generalized Riemann hypothesis is open one. 